\title{
Research of e-Learning Platform based on Open Source Framework
}

\author{
Na Zhang ${ }^{1, a}$, Jia Wang ${ }^{2, b}$ \\ ${ }^{1}$ Department of Software Engineering, Dalian Neusoft University of Information, \\ Dalian, 116023, China \\ ${ }^{2}$ Education Resources Development and Training Center, Dalian Neusoft University of Information, \\ Dalian, 116023, China \\ aemail: zhangna@neusoft.edu.cn, bemail: wangjia@neusoft.edu.cn
}

Keywords: e-Learning; Java EE; NEO Framework

\begin{abstract}
The gradual development of Java EE architecture promotes the research and development of e-Learning platform. The research status and future development trend of e-Learning platform are analyzed, and the NEO Framework is proposed. NEO Framework based on Java EE architecture, many open source frameworks and MVC design pattern is a new combination framework, which mainly uses the techniques of FreeMarker, Struts nd Spring etc. It is very suitable for the development of e-Learning platform.
\end{abstract}

\section{Introduction}

With the popularity of the network and the rapid expansion of Internet, online learning known as e-Learning, are being used more and more widely in the distance education, group training, teaching in colleges and universities. The modern university education is more and more reliance on e-Learning model because of the growing in enrollment scale, scattered campus, the development of electronic teaching.

Computer into the field of education has experienced two stages. The first stage is called the Computer Aided Instruction (CAI), namely teachers demonstrate the partial knowledge point to students by computer in order to display and understand easily. This form only uses the computer as a teaching aid, in fact, did not cause the change of educational theory and teaching mode. The second stage is called Digital Campus, created by multimedia technology and virtual reality technology after the birth of the Internet. It is the extension of the real campus. As a basic platform of teaching resources and teaching process informatization, e-Learning platform is the core application and one of the most important applications in Digital Campus. E-learning platform creates free and wide environment for students, provides wealthy resources, expands the teaching time and space, makes a transition from the traditional teaching mode with teachers as center to the autonomous learning mode with students as center.

In this new situation, in order to solve the problems in e-Learning platform development, a set of scientific software development framework should be proposed to meet the needs of the growing of enterprise web application [1].

In this paper a new development framework is put forward, that is, NEO Framework. It combined Java EE architecture and MVC thought and obtained the great improvement in development efficiency. NEO Framework provided very well encapsulation and abstraction and was easy to learn. Because of its clearly hierarchical thought, the codes based on NEO Framework are easily extensible and maintainable.

\section{Overview of e-Learning}

E-learning refers to the learning and teaching activities through the Internet and other digital medium. It makes full use of modern information technology, new communication mechanism and abundant learning environment to achieve a new way of learning. This kind of style will change the 
teachers' role and the relationship between teachers and students, so as to fundamentally change the teaching structure and the nature of education.

The origin of e-Learning is in the United States. In China, although e-Learning started late, but there is a hug market potential [2]. For individuals, lifelong learning is a necessary condition for the success of modern people. Therefore, e-Learning based on Internet and information technology has been paid attention by enterprises and academia. The research about e-Learning not only meets the needs of the network teaching in Colleges and universities, and also brings a high practical value for the enterprise internal training.

E-learning has the following characteristics:

- Information technology is the basis of e-Learning

- E-Learning is not restricted by time and space

- E-Learning is suitable for a variety of learning groups

- E- Learning is an effective way to learn

- E-Learning is generally low cost to the education provider

Through e-Learning, students can browse the teaching contents, finish the homework and participate in tests and so on; teachers can announce messages, publish papers, upload courseware and organize discussion and so on. Teachers and students are in the relative separation from time to space, the students are based on self-study, supplemented by teachers. In the near future, e-Learning will become an important part of school education.

\section{Design of NEO Framework}

Java EE is the abbreviation of the Java Platform Enterprise Edition; it is a distributed enterprise application development technical architecture and an open and standard enterprise development and deployment platform. Traditional database server mode has been unable to meet the urgent requirement on web application rapid development, expansion and reorganization of resources. At this moment, web application of distributed process based on Java EE architecture arises. It packages business process logic, improves the reusability and scalability of the program [3].

Java EE defines four layer architecture, including client layer, web layer, business layer and enterprise information system layer. Each layer provides a certain class of functions for application. Users request is usually transmitted among multilayers, finally is responded. The level model in Java EE separate business logic from user interface of application, thus ensuring the developers can focus on the analysis, planning and design of core application business and quickly establish core business function of application system.

MVC is the abbreviation of Model-View-Controller; it is the design pattern of Java EE and is mandatory to separate the data, data representation and user actions of application. User view module is to generate the returned client pages according to the result of business logic module. Business logic module is to process operation flow and develop business rules. Controller module is to implement the collaborative work of view and model.

Combining Java EE layered thought and the MVC design pattern, NEO Framework includes three layer structures, namely presentation layer, business logic layer and data layer. Presentation layer is subdivided into page resource layer and page template layer. Business logic layer is subdivided into control layer, business processing layer and data persistence layer. The architecture of the NEO Framework is illustrated in Figure 1.

A lot of components and technologies are used in the NEO Framework. In the presentation layer, FreeMarker is used to generate the final pages. FreeMarker is a template engine and a general tool to output text according to template. FreeMarker as an alternative to JSP, can be used to separate visual design and application logic in MVC pattern. Therefore, in the NEO Framework, the design and implementation of the presentation layer is more hierarchical because of the use of FreeMarker. 


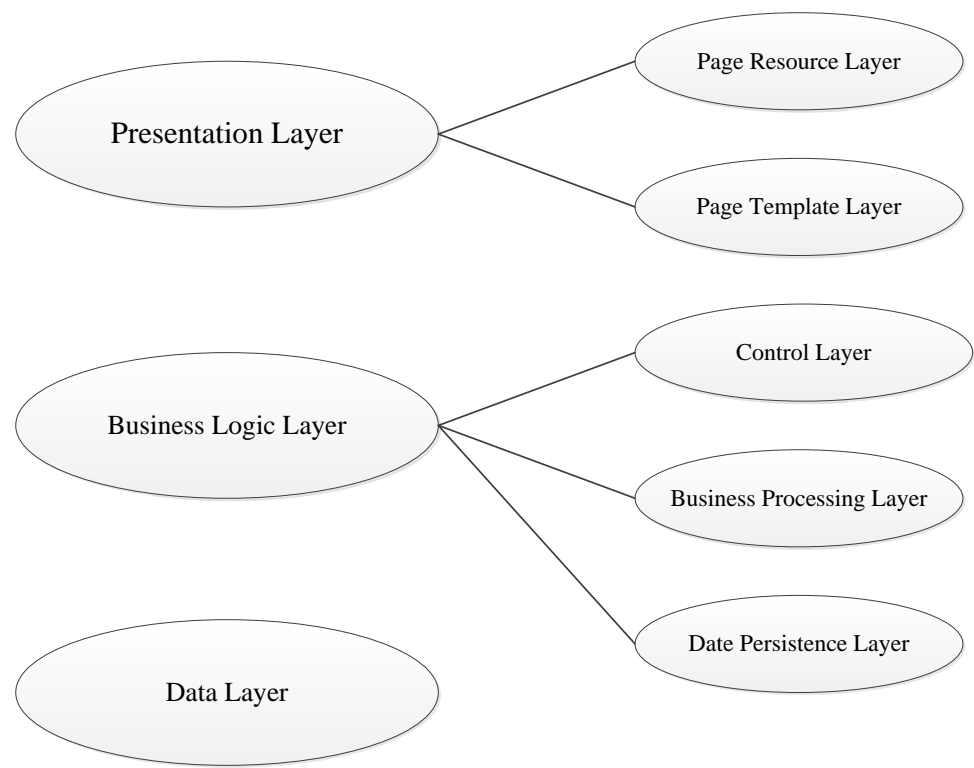

Fig.1. Architecture of NEO Framework

In the control layer, Struts is used, which is a powerful MVC Web framework. One of the reasons for the selection of Struts is the structural and hierarchical NEO Framework. On the other hand, Struts will help reducing coding and allow developers to more focus on the business logic and modeling. And Struts supports multiple view technologies, including FreeMarker.

In the business processing layer, Spring Framework is used, which is a lightweight Java EE application framework. The core of Spring is a lightweight container, which implements the inversion of control (IoC) pattern. IoC pattern helps to simplify coding, separate the business logic from the infrastructure, and make application more easier to maintain [4].

In the data persistence layer, Spring JdbcTemplate is used, which is a good package for JDBC, by providing the appropriate template and auxiliary classes to reduce the complexity of the JDBC operation. And because of the isolation design of Spring, Spring JdbcTemplate can be independently used without Spring Context.

\section{Analysis of NEO Framework}

In Java EE architecture, presentation logic layer and business logic layer are both in the application server, which are implemented by Java EE standard components, such as JSP, servlet, EJB etc. [5] In the NEO Framework, although there are also presentation layer and business logic layer, but the means of the implementation have been improved, Figure 2 is the technique architecture of NEO Framework.

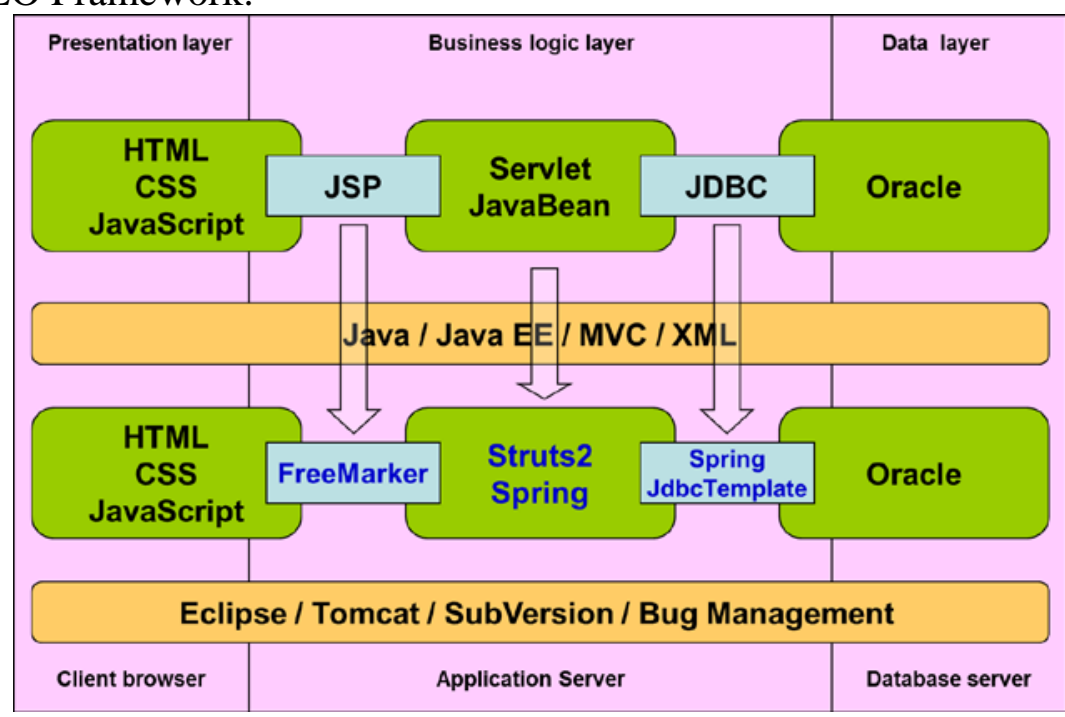

Fig.2. Technique architecture of NEO Framework 
Compared with traditional Java EE architecture, there are several improvements in NEO Framework as follows:

- Division of the page is clearer. The page resource layer is composed of HTML, CSS, JavaScript, but they play different roles. So it provides a more reliable possibility for future project modifications and codes reuse.

- Page template engine is used. The use of FreeMarker greatly improves the previous confusion of page code, data objects and Java code caused by JSP.

- Struts is instead of Servlet. Struts not only can use the Servlet processing function, but also plays the core function.

- Spring intervenes in the business process. The Spring framework is more simple and more lightweight than Java EE. The NEO Framework makes full use of Spring IoC, which makes the business process more simple and convenient.

- JdbcTemplate is introduced in the data persistence layer. This leaves out a lot of repeated codes, avoids possible database connection leaks exceptions, and improves the development efficiency.

\section{Conclusion}

Based on the analysis of the e-Learning platform and Java EE architecture as the foundation, NEO Framework was proposed. It provides developers with an easy-to-use model. Using it to develop e-Learning platform, developers can code without understand low-level APIs. NEO Framework provides a clear structure for a project which makes it easier for others to join the project development.

\section{References}

[1] Guohua Zuo. Research on Distributed Enterprise Web application based on J2EE Architecture [J], Wuhan : Huazhong University of Science and Technology, 2005.

[2] Jinbing Zhang, Shengquan Yu. Development Trend of e-Learning in China. Chinese Distance Education Journal (Information Edition), 2003 24(5) 120-134.

[3] Hui Liu, Yuchen Li. Research on Distributed Web Application based on J2EE Architecture. Computer Application Research, 200315 (9) 47-49.

[4] Jun Mu, Li Lv. The Use of Lightweight Framework for J2EE Application Development. Small and Micro Computer System, 2006 27(6) 1149-1152.

[5] Wei Li, Junzhou Luo, Jiuxin Cao. An Integrated Framework and Application of Network Education System based on J2EE. Computer Research and Development, 2006 43(8) 1354-1360. 